\title{
USING THE CORRECT SIZE OF AMPLATZ SHEATH DURING PERCUTANEOUS NEPHROLITHOTOMY - A NOVEL METHOD.
}

\section{Rahul Goel*}

\section{Department of Surgery, Muzaffarnagar Medical College, Muzaffarnagar. *Corresponding Author}

ABSTRACT PURPOSE- To choose appropriate size Amplatz sheath, during percutaneous nephrolithotomy (PCNL) based on retrograde nephrogram (RGP).

MATERIAL AND METHODS -Standard PCNL , in prone position, was performed in 20 patients. All patients had placement of Amplatz sheath based on retrograde nephrostogram study, all patients after surgery had nephrostomy tube for draining collecting system. The preoperative and postoperative renal parameters ,hemoglobin ,mean operating time and mean hospitalisation stay was measured.

RESULTS- There was no significant difference in the pre and postoperative hemoglobin and creatinine levels . Median operating time was 60.5 mins (45 to 98 mins).The median hospitalisation stay was 4.6 days( 3 to 7 days).There was no intraoperative pelvic wall or infundibular injury.

CONCLUSION- Choosing correct size Amplatz sheath decreases the incidence of bleeding and renal function impairment.

\section{KEYWORDS :}

\section{INTRODUCTION-}

The art of PCNL has evolved in terms of technique over years ,to improve its outcome and decrease complications.(1-3), it has advanced in terms of small track size, reducing radiation and patients positioning, PCNL in recent years has been performed by using small size Amplatz sheaths, to avoid comlications and reduce morbidities. There is little literature evidence in selecting appropriate size Amplatz sheath in PCNL.

The present study discusses a technique which helps to determine the correct size of Amplatz during percutaneous nephrolithotomy (PCNL).

TABLE 1 . Some demographic characteristics of the patients

\begin{tabular}{|l|l|}
\hline DATA & Values \\
\hline Mean age in years ( Range) & $44.5(22-58)$ \\
\hline SEX & \\
\hline \multicolumn{1}{|c|}{ Male } & 16 \\
\hline \multicolumn{1}{|c|}{ Female } & 04 \\
\hline Median stone size (range) in cms & $2.56(2-4)$ \\
\hline Targeted Calyx & \\
\hline Middle & 5 \\
\hline Inferior & 15 \\
\hline Laterality & \\
\hline Right & 09 \\
\hline Left & 11 \\
\hline
\end{tabular}

Table 2. Results

\begin{tabular}{|l|l|}
\hline Data & Number of cases \\
\hline Amplatz sheath size & \\
\hline $28 \mathrm{Fr}$ & 06 \\
\hline $26 \mathrm{Fr}$ & 07 \\
\hline $24 \mathrm{Fr}$ & 04 \\
\hline $22 \mathrm{Fr}$ & 03 \\
\hline Mean operative time (mins) & $74+-22.3(\mathrm{range} 45-135)$ \\
\hline Hemoglobin $(\mathrm{Hb})$ Levels & \\
\hline Preoperative & $12.2+-0.8 \mathrm{~g} / \mathrm{dl}$ \\
\hline Postoperative & $11.8+-0.6 \mathrm{~g} / \mathrm{dl}$ \\
\hline Median hospital stay & 3.4 days $(3-7$ days) \\
\hline
\end{tabular}

\section{MATERIAL AND METHODS}

The study was done between April 2015 and April 2017, 20 patients were included with only pelvic stone

in our protocol. The mean stone size, and other patients demographics are given in Table 1. All patients had standard PCNL with stent and nephrostomy in spinal anaesthesia.
After placement of ureteric catheter cystoscopically retrograde pyelogram (RGP) was done by instilling diluted contrast. The size of the desired calyceal infundibulum was measured, as the shortest distance between the edges of the fluoroscopic infundibular image nearer to pelvis (Figure 2), Then serially starting from small to large size Amplatz sheath were placed over the back of patient (patient in prone position) and fluoroscopic match was obtained between infundibular diameter and appropriate Amplatz sheath (Figure 1). Calyx was entered under fluoroscopic guidance, tract was serially dilated up to a predetermined Amplatz size ,decided by RGP and then appropriate Amplatz sheath was placed.

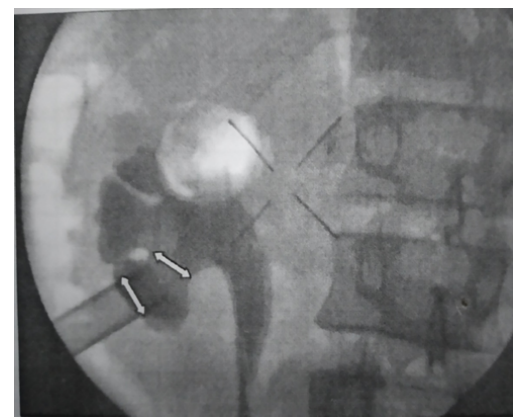

Figure 1 - 28 Fr Amplatz match

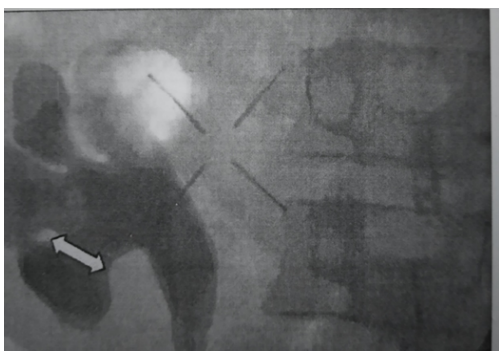

Figure 2. - Infundibular diameter (arrow)

Stone was cleared and a DJ stent was placed, Nephrostomy tube of 2Fr less was placed of the size of Amplatz sheath used, nephrostomy tube was kept and removed after 24 hours, foleys per urethral catheter removed the next day .The renal parameters and hemoglobin levels were checked after 6 hours postoperatively.

Statistical analysis

The comparison of mean values of continuous variables was 
done using the student's t-test .If the $p$ value was $<0.05$, the result was considered statistically significant.

\section{RESULTS}

Median age of patients was 44.5 yrs ( range ,22-58yrs) and median stone diameter was $2.56 \mathrm{cms}$ ( range $2-5 \mathrm{cms}$ ). Mean operative time was $74+-22.3$ mins(range , $45-135$ mins). All patients had a single tract either middle or lower calyx. Of the 20 patients $, 28 \mathrm{Fr}(n=20), 26 \mathrm{Fr}(n=6), 24 \mathrm{Fr}(n=4)$, and $22 \mathrm{Fr}$ $(n=3)$ Amplatz sheaths were used for indicated number of patients.

The mean preoperative and postoperative hemoglobin $(\mathrm{Hb})$ levels in all 20 patients who underwent the procedure was $12.2+-0.8 \mathrm{~g} / \mathrm{dl}$ and $11.8+-0.6 \mathrm{~g} / \mathrm{dl}$, respectively. There was no statistically significant difference between the pre and post operative hemoglobin values $(\mathrm{p}>0.3)$.

There was no urothelial injury to infundibulum or pelvis, the median hospital stay was 3.4days (3-7days). One patient post operatively developed sepsis but recovered, two patients had haematuria for $>24$ hrs post operatively via foleys catheter, with no significant drop in hemoglobin levels (Table 2).

\section{DISCUSSION}

Bleeding is a dreaded complication of PCNL, depending on correct choice of access site, dilatation, size of Amplatz sheath, size of stone and the presence of infection. There are many studies describing exit strategy in PCNL to reduce complications ,but not many studies regarding entry strategy for the same.

Desai et $\alpha l(14)$ in there study concluded that tubeless PCNL procedure is associated with less morbidity, but there was no mention of the tract size and the method used to choose Amplatz in the study .

Karakose et al(9) did a study comparing small vs large size Amplatz ,and have shown less complications with using small Amplatz sheath, but they also did not show how to choose an Amplatz sheath for a particular case. So to our knowledge this study is the first of its kind.

In this study size of Amplatz sheath was chosen depending upon the infundibular diameter, and no case was over dilated beyond this diameter. All patients were punctured in calyx in prone position so all calyces punctured were posterior, and no significant bleeding was encountered in any case.

An important part in dilatation is the inherent elasticity and expandability of the calyces, at least to some extent ,compensates for some mismatch between Amplatz sheath and the size of calyx on fluoroscopy.

The argument of choosing a small tract with small nephroscope can be used to avoid complications, but with large stone burden and well dilated calyx and infundibulam using a small tract would lead to prolonged surgical time and prone to more sepsis due to increased intra pelvic pressure seen in small size tract.

From our study one could avoid a narrow calyx and choose an alternate site of entry to be used for stone clearance, though this study is limited by its small sample size and non randomisation.

In conclusion it is well known small size Amplatz sheath have less, bleeding ,renal impairment and post operative pain in comparison to large size of Amplatz sheath,we Suggest the size of Amplatz sheath to be decided using our technique of using RGP (retrograde pyelograms ) as the guide to dilate .Further study comparing standard PCNL and our method of choosing Amplatz size depending on RGP are needed to reinforce our findings.

\section{REFERENCES}

1. CormioL, Preminger G, Saussine C, Buchholz NP, Zhang X, Walfridsson H et al. Nephrostomy in percutaneous nephrolithotomy (PCNL):does nephrostomy tube size matters? Result from the Global PCNL study from The clinical Research Office Endourology Society. World J Urol 2013:31:1563-8

2. Fernstrom I, Johansson B. Percutaneous pyelolithotomy .A new ex-traction technique.Scand J Urol Nephrol 1976;10:257-9

3. De Sio M, Autorino R, Quattrone C, Giugliano F,Balsamo R, D'Armiento M. Choosing the nephrostomy size after percutaneous nephrolithotomy. World J Urol2011;29:707-11

4. Desai MR, Kukreja RA, Desai MM, Mhaskar SS,Wani KA, Patel SH,et al. A prospective randomised comparison of type of nephrostomy drainage following percutaneous nephrolithotomy :large bore vs small bore vs tubeless JUrol $2004 ; 172: 565-7$

5. Shah HN ,Sodha HS, Khandkar AA, Kharodawala S, Hegde SS, Bansal MB. A randomised trail evaluating type of nephrostomy drainage after percutaneous nephrolithotomy :small bore vs tube less .J Endourol 2008:22:1433-9

6. Crook TJ ,Lockyer CR, SR, Walmsley BH. A randomised control trial of nephrostomy placement versus tubeless percutaneous nephrolithotomy .J Urol2008; 180:612-4

7. Limb J, Bellman GC. Tubeless percutaneous renal surgery :Review of first 112 patients .Urology 2002:59:527-31

8. Crook TJ ,Lockyer CR, Keoghane SR, Walmsley BH Totally tubeless percutaneous nephrolithotomy .J Endourol2008;22:267-71

9. Karakose A, Aydogdu O, Atesci YZ. Does the use of smaller Amplatz sheath size reduce complication rates in percutaneous nephrolithotomy ? Urol 2014;11:1752-6 